\title{
CAPITAL HUMANO Y
} FACTORES SOCIOLÓGICOS COMO DETERMINANTES DEL EMPRENDIMIENTO

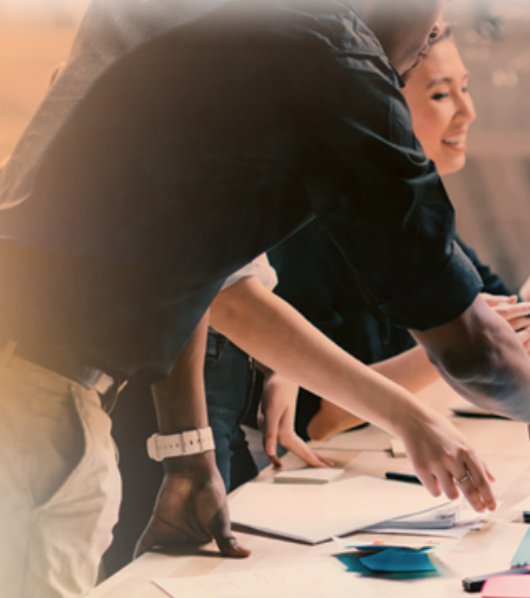

Human cappitic

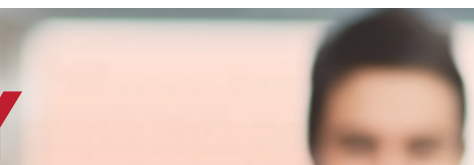




\section{ग) INTRODUCCIÓN}

En el informe sobre el crecimiento inclusivo del Foro Económico Mundial (World Bank, 2014), se identifican las características estructurales e institucionales de los países y cómo, por medio de una serie de pilares -considerados necesarios-, las economías buscan incrementar el crecimiento y la equidad para sus habitantes, haciendo énfasis en la educación y el emprendimiento como ejes primordiales para el incremento y la mejora en los niveles de vida de un país. De acuerdo con el Índice de Condiciones Sistémicas para el Emprendimiento Dinámico (Kantis, Federico, y García, 2014), los emprendedores dinámicos son los que tienen mayor capacidad para crear empleos de calidad y ayudar a diversificar la estructura productiva en una región, logrando convertirse en pocos años en pymes competitivas con potencial de seguir creciendo, cuya base es la diferenciación y la innovación, razón por la cual cada vez son más los países que están interesados en promover el surgimiento de estos emprendimientos.

El Estado costarricense ha presentado avances significativos en el apoyo al emprendimiento y la importancia que posee en la economía del país, tanto desde los Ministerios encargados como desde la academia que impulsa programas y proyectos de emprendedurismo, operacionalizando los instrumentos derivados de la Ley PYME $^{1}$ y otras iniciativas de apoyo al desarrollo empresarial. Estos esfuerzos no se han visto acompañados por un cuerpo de investigación científica que permita evaluar en profundidad los determinantes de la actividad emprendedora en el país, con el objetivo de dar recomendaciones que permitan incrementar el nivel de alineación entre las características del emprendedor en

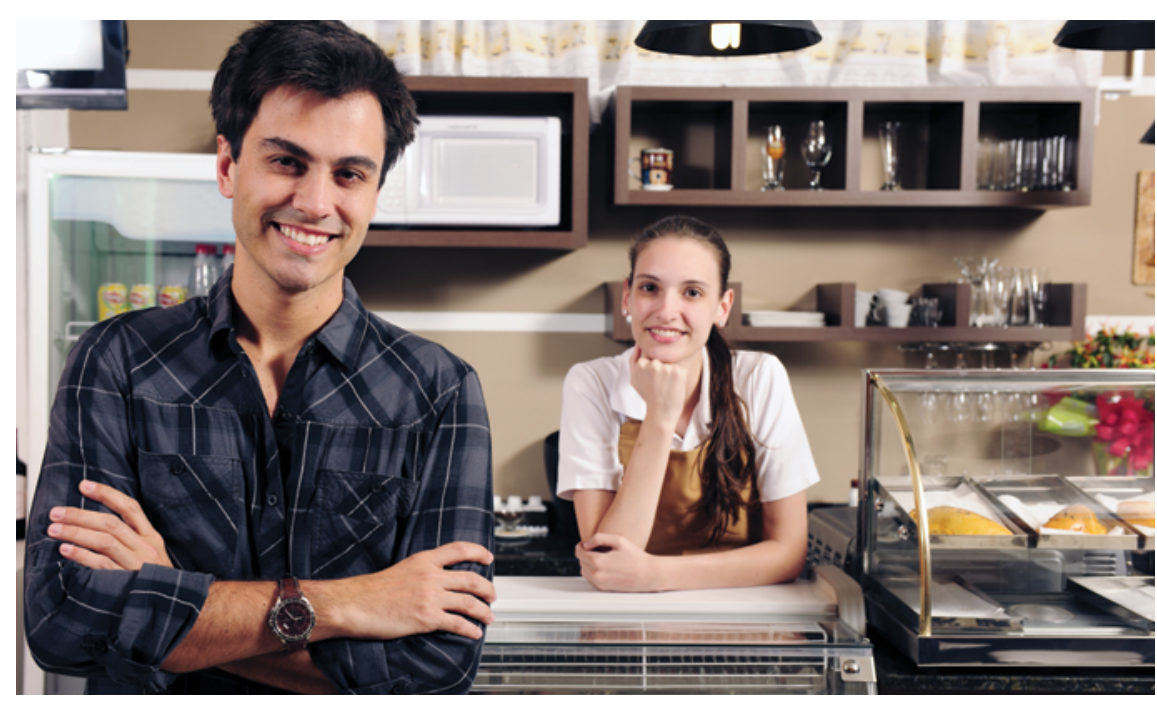

Costa Rica y el diseño de políticas de apoyo asociadas al emprendimiento.

El emprendimiento ha demostrado que no es únicamente una actividad personal por parte de quien lo desarrolla, sino que, además, es un generador social y económico, ya que impulsa la innovación, mejora la competitividad industrial, incrementa la productividad a través del uso de tecnologías y, consecuentemente, permite alcanzar niveles superiores de bienestar y desarrollo a nivel regional o nacional (Lafuente, Szerb y Acs, 2016). Para el caso costarricense, se observa cómo las externalidades positivas derivadas del aprendizaje social, sean estas la adopción de nuevas tecnologías, creación de nuevos empleos y el pago de impuestos, aumentan la competitividad del país, estimulan la innovación y el emprendimiento, y fortalecen estrategias a medio plazo para la incorporación a mercados competitivos (PEN, 2014; Ministerio de Ciencia Tecnología y Telecomunicaciones, 2007).

El artículo busca contribuir al conocimiento por medio de dos objetivos, i) analizar el capital humano-formación-y su relación con la decisión emprendedora y el desempeño de las nuevas empresas, ii) explicar si las habilidades percibidas -autoconfian$z a-$ influyen en la decisión emprendedora y el desempeño de las nuevas empresas, de forma tal que la información acá generada sea útil para el diseño de política pública en la materia.

Para alcanzar estos objetivos se propone un enfoque cuantitativo que busca analizar los efectos sobre la actividad emprendedora (creación de empresas) de una serie de variables relacionadas como: la formación, la experiencia laboral, la autoconfianza, los ejemplos emprendedores y el miedo al fracaso. La aplicación empírica emplea la base de datos generada por el Global Entrepreneurship Monitor (GEM) para Costa Rica en el año 2014.

El resto del documento está organizado de la siguiente manera. La revisión teórica se presenta en la sección 2. En la sección 3 se expone la metodología de investigación. La discusión de los resultados se ofrece en la sección 4, y las conclusiones obtenidas se muestran en la sección 5 . 


\section{REVISIÓN DE LA LITERATURA Y FORMULACIÓN DE HIPÓTESIS}

La teoría del capital humano surge originalmente como reacción a la necesidad por estudiar el valor de la formación, debido a que las personas poseen diferentes conocimientos y habilidades que tienen un valor económico (Becker, 2003). Becker formuló la teoría de las inversiones en capital humano, basándose en la evidencia de que las personas altamente educadas y capacitadas casi siempre tienden a ganar más que otras, siendo una teoría para entender mejor la contribución del conocimiento que poseen las personas respecto a otros factores de producción, como el capital (Becker, 2003; Marvel, Davis y Sproul, 2014).

El capital humano puede dividirse en general y específico. Por capital humano general se comprende el conocimiento adquirido a través de la educación formal y experiencia profesional; y el específico envuelve las capacidades de las personas, que pueden ser aplicadas directamente a un trabajo o dentro de una empresa, que son aprendidas previamente en entornos organizacionales y que resultan de la acumulación de experiencia en una organización (Estrin, Mickiewicz y Stephan, 2016; Debrulle, Maes y Sels, 2013; Westhead, Ucbasaran y Wright, 2009).

Los emprendedores con mayores niveles de capital humano específico en el momento de la puesta en marcha de la empresa tienen expectativas más realistas, están más satisfechos con el rendimiento financiero, o utilidad no monetaria derivada del negocio, y buscan obtener un bienestar psicológico (por ejemplo, tiempo de ocio). Entre los emprendedores con alto nivel de capital humano general, sus expectativas empresariales hacen que sea más difícil lograr la satisfacción en la gestión emprendedora (Becker, 1993; Marvel y Lumpkin, 2007; Marvel et al., 2014).

\section{Actitud emprendedora y el capital humano formal}

Las actitudes emprendedoras son el grado en que las personas piensan que hay buenas oportunidades para comenzar un negocio, la asignación de un estatus alto y reconocimiento, además del nivel de riesgo que la persona está dispuesta a soportar, la percepción que las personas tienen de sus propias habilidades y conocimientos, así como la experiencia en la creación de un negocio (Amorós, 2011). Profundizando en el capital humano formal, se muestra la teoría relacionada con la formación y experiencia laboral.

Diversas definiciones literarias establecen que la formación, compuesta por conocimientos y habilidade s, son el resultado de inversiones en capital humano, como la formación y la experiencia laboral, donde una educación emprendedora produce más y mejores emprendedores que los producidos en el pasado, siendo la educación, experiencia, conocimiento y habilidades, recursos críticos para el éxito emprendedor. El espíritu emprendedor como campo de estudio refleja importantes contribuciones económicas en términos de empleo y creación de riqueza, donde los emprendedores poseen atributos que incluyen la voluntad de tomar riesgos calculados, propensión a innovar y determinación a tener éxito (Unger, Rauch, Frese y Rosenbusch, 2011; Bell, Callaghan, Demick y Scharf, 2004).

La educación formal emerge del capital humano general, donde la experiencia en la gestión es fuente de inteligencia y habilidades genéricas, pues forja un conocimiento individual para mejorar el razonamiento cognitivo, en el que prevalece el procesamiento y las habilidades para resolver problemas, ya que refleja una necesidad de motivación para el logro, aumenta el com- promiso y el esfuerzo dedicado a la búsque$\mathrm{da}$, por lo que se espera que las personas con mayores niveles de educación formal sean más receptivas a las nuevas ideas y al cambio (Debrulle et al., 2013).

El fomento de la educación formal en el tema de espíritu emprendedor y el beneficio económico a la sociedad produce un incremento de emprendedores en la población, quienes estimulan los niveles de actividades económicas. La educación formal proporciona las habilidades cognitivas necesarias para adaptarse a los cambios del entorno, donde los emprendedores altamente educados pueden estar en mejores condiciones a la solución de problemas complejos, aprovechando sus conocimientos y contactos sociales para adquirir, identificar y explotar las oportunidades de negocio (Van Praag, van Witteloostuijn y van der Sluis, 2013; Honjo, Kato y Okamuro, 2013; Jayawarna, Jones y Macpherson, 2014; O’Connor, 2013).

El resumen de la discusión literaria sobre esta variable se puede observar en el apéndice 1 , donde se aprecian los trabajos realizados por los investigadores y su contribución al tema, en los cuales se muestran los objetivos planteados, el método utilizado y hallazgos clave. De estos argumentos y evidencia surge la primera hipótesis propuesta.

H1: El capital humano formal del individuo, en términos de educación, tiene un efecto positivo sobre la decisión de crear una empresa.

\section{Actitud emprendedora y el capital humano informal}

La autoconfianza - self confidence- representa el conjunto de habilidades emprendedoras relacionadas con el nivel de interés que puede tener una persona y la decisión de convertirse en un emprendedor, es considerada como un activo importante que $\gg$

\section{Este artículo analiza cómo distintas formas de capital humano afectan la decisión emprendedora}


b) posee un individuo y que ayuda a lograr el éxito personal, siendo, además un proceso cognitivo en el que las personas construyen creencias acerca de su nivel de capacidad para el logro (Kirkwood, 2009; Gelaidan y Abdullateef, 2017; Pihie y Bagheri, 2011).

Se asocia, asimismo, con el coraje necesario para asegurar las competencias, valores y metas, y destaca atributos como: la creencia en logros positivos, tales como el optimismo y la autoafirmación; la persistencia, entre ellos la resiliencia, visión, previsión, fijación de metas; y autoconciencia, niveles de ansiedad, excitación e intuición. La percepción de sí mismo y el self-confidence de una persona competente son diferenciadores de los individuos que han creado sus propios emprendimientos de aquellos que no lo han hecho (Karabay, Akyüz, y Elçi, 2016; Shook, Priem y McGee, 2003).

Bandura (1999) formula una definición de self-confidence a través de la teoría cognitiva social que incluye el entorno social y los elementos cognitivos, así como la conducta misma, conocida como Teoría Social Cognitiva (SCT), donde el self-confidence es un ajuste personal o una creencia en qué tan bien se puede ejecutar la acción requerida para lidiar con situaciones prospectivas, además de la descripción del concepto de confianza en sí mismo, y la autopercepción de sus propias capacidades, enfatizando en la importancia de la motivación, el refuerzo y los factores de experiencias pasadas para establecer y fomentar la confianza de un individuo (Karabay et al., 2016; Indrawati, Salim, Djumahir y Djawahir; 2015).

Investigaciones previas sobre el espíritu empresarial indican que la autoconfianza representa un indicador muy objetivo sobre el desempeño empresarial, que está conectado al reconocimiento de oportunidades, de la intención y la decisión de hacer una carrera emprendedora (e.g.,
Lafuente, Vaillant, y Rialp, 2007; Vaillant y Lafuente, 2009). A más altos niveles de auto-confianza emprendedora, mayor vinculación con las intenciones de iniciar una nueva empresa, reforzando así el argumento de que la auto-confianza emprendedora es un antecedente importante para la acción, al igual que la educación y las experiencias que se pueden relacionar con los resultados emprendedores. Del mismo modo lo es la capacidad de una persona para obtener un resultado deseado, el cual busca explicar por qué los individuos con capacidades similares tienen un desempeño diferente en una tarea, pues, al tener una alta autoconfianza, ejercerá más esfuerzo por un mayor período de tiempo para realizar una tarea, debido a que persiste a través de reveses, establece y acepta metas más altas, así como el desarrollo de mejores planes y estrategias para conseguir la meta (Arora, Haynie y Laurence, 2013; Cardon y Kirk, 2015).

Sin embargo, se ha documentado que, en muchas ocasiones, las personas con altos niveles de capital humano no materializan sus proyectos emprendedores. En este sentido, Benabou y Tirole (2002) sugieren que la auto-confianza puede actuar como antídoto

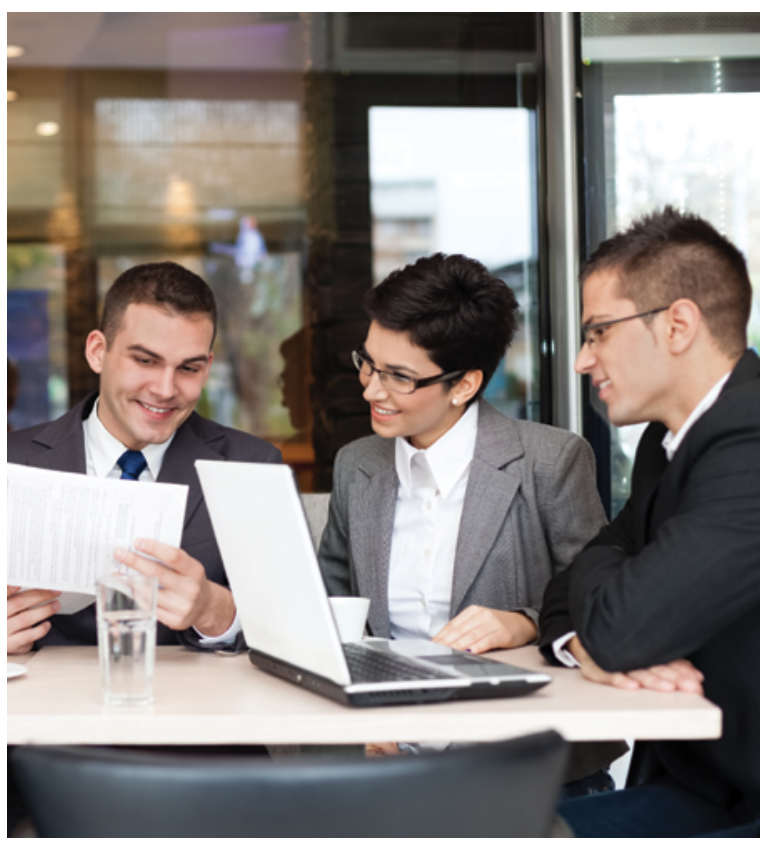

para la baja tasa de iniciación emprendedora entre personas con alto capital humano. $\mathrm{La}$ auto-confianza en las habilidades empresariales afecta la respuesta de los individuos ante cambios en los entornos de mercado donde se desenvuelve, lo que favorece la acción emprendedora. Además, la explotación de ideas emprendedoras implica la asunción de riesgo -principalmente financiero- que requiere altos niveles de confianza tanto en las habilidades propias como en el capital humano que se posee para llevar a cabo cualquier iniciativa emprendedora (Henley, 2007; Baron, 2008; Bayon, Lafuente y Vaillant, 2016). En el apéndice 2 se muestra el resumen de las variables investigadas; de estos argumentos y evidencia surge la segunda hipótesis de este trabajo.

H2: El capital humano informal del individuo, en términos de habilidades percibidas -autoconfianza- tiene un efecto positivo sobre la decisión de crear una empresa.

\section{MUESTRA, DEFINICIÓN DE VARIABLES Y MÉTODO DE ANÁLISIS}

En este estudio se emplea un modelo de regresión logística como técnica de análisis con el objetivo de dar respuesta a los objetivos planteados. Las variables de estudio fueron medidas a través de la encuesta aplicada por el GEM Costa Rica en el año 2014, utilizando la Encuesta a la Población Adulta -EPA- aplicada a hombres y mujeres con una edad comprendida entre 18 y 65 años, quienes fueron entrevistados telefónicamente, cara a cara o una combinación de ambos. La base de datos inicial contiene respuestas para 2058 costarricenses localizados en todo el país, pero, una vez desestimadas aquellas observaciones que carecían de información completa para todas las variables, el tamaño de la muestra final empleada en esta 
investigación es de 2011 personas cuyo emprendimiento es de reciente creación. Es un estudio transversal, debido a que los datos se tomaron en un solo momento de tiempo -entre mayo y agosto de 2014- y de diseño cuantitativo. Las variables seleccionadas responden al interés de los investigadores en medir su impacto en el emprendimiento en Costa Rica.

Para la revisión teórica, se analizaron diversos artículos relacionados con las variables de estudio, algunas de ellas fueron agrupadas en tablas unificando las investigaciones realizadas, las cuales se encuentran en la sección de Apéndice.

A su vez, se definieron las variables investigadas ubicadas en la tabla 1 , donde el GEM describe al nuevo emprendedor como aquella empresa que tiene al menos cuarenta y dos meses de haber sido creada; un $3,8 \%$ de los encuestados identifican su emprendimiento dentro de esta categoría. Por otro lado, se considera como emprendedor naciente aquel cuya puesta en marcha tiene un año de estar explorando el mercado, y corresponde a un 7,6\% de quienes respondieron la encuesta.

Se observa que un $41,42 \%$ de los emprendedores tiene un ejemplo emprendedor (role model). Tal y como indican Lafuente, Vaillant, y Rialp (2007) y Bosma, Hessels, Schutjens, van Praag, y Verheul (2012), la presencia de un ejemplo emprendedor puede emular y estimular a otras personas para que tomen ciertas decisiones. En contraposición y en un porcentaje menor, el 39,28\% de los encuestados posee temor al fracaso; según lo manifestado por Edelman, Manolova, Shirokova y Tsukanova (2016), se asocia la actividad emprendedora con el temor al fracaso, caracterizado por evitar ciertas acciones, haciendo que los individuos pospongan tanto la planificación como la ejecución.

En cuanto a la autoconfianza como habilidad percibida, el $59,77 \%$ de las dos mil personas encuestadas consideran tener esta habilidad; Kirkwood (2009) la define como un activo importante que posee un

Tabla 1: Estadísticos descriptivos

\begin{tabular}{|c|c|c|}
\hline & Promedio & $\begin{array}{l}\text { Desviación } \\
\text { estándar }\end{array}$ \\
\hline Nuevo emprendedor (baby business) & 0,0383 & 0,1919 \\
\hline Emprendedor naciente & 0,0761 & 0,2652 \\
\hline Ejemplo emprendedor (Role model) & 0,4142 & 0,4927 \\
\hline Miedo al fracaso & 0,3928 & 0,4885 \\
\hline Habilidades percibidas & 0,5977 & 0,4905 \\
\hline Género & 0,4779 & 0,4996 \\
\hline Edad & 37,2163 & 13,2858 \\
\hline Educación primaria & 0,2516 & 0,4340 \\
\hline Educación secundaria & 0,3401 & 0,4739 \\
\hline $\begin{array}{l}\text { Educación post secundaria (formación } \\
\text { vocacional + universidad) }\end{array}$ & 0,4083 & 0,6645 \\
\hline Localización: San José & 0,3366 & 0,4727 \\
\hline Localización: Alajuela & 0,1919 & 0,3939 \\
\hline Localización: Cartago & 0,1183 & 0,3231 \\
\hline Localización: Heredia & 0,1099 & 0,3128 \\
\hline Localización: Guanacaste & 0,0721 & 0,2587 \\
\hline Localización: Limón & 0,0840 & 0,2775 \\
\hline Localización: Puntarenas & 0,0870 & 0,2819 \\
\hline
\end{tabular}

Número de observaciones en la muestra: 2011 individuos.

individuo y cuya decisión de convertirse en un emprendedor le ayudará a lograr el éxito personal. Se determina, además, que el $40,83 \%$ de las personas que poseen una educación vocacional/universitaria emprenden más en comparación con personas cuyo nivel educativo es inferior. Según Unger et al. (2011) y Kakouris (2016), la formación produce más y mejores emprendedores que los producidos en el pasado, debido al uso de pedagogías innovadoras que se reflejan en el usuario final.

En cuanto a las variables de control, iniciando con el género (Driga, Lafuente y Vaillant, 2009), el 47,80\% son hombres emprendedores, según lo expresa la OCDE (2004), por lo que el género es un factor importante que explica los diferentes niveles de propensión de los individuos hacia el emprendimiento. A su vez, se establece que la edad promedio de los emprendedores encuestados es de 37 años, sobrepasando por poco el límite de edad estudiado por Shane (1996); la composición de la población y nivel de emprendimiento se da en personas en edades entre los 25 a 34 años.

Finalmente, para la variable localización, se observa que el emprendimiento es más intenso en zonas predominante urbanas (San José, Cartago, Alajuela y Heredia) 》 
d) donde el porcentaje de emprendedores oscila entre el $12 \%$ y el $34 \%$, mientras que en zonas con mayores tasas de ruralidad este porcentaje se encuentra entre el $9 \%$ y el $11 \%$. Este resultado es similar al reportado por Vaillant y Lafuente (2007).

La ecuación 1 busca establecer la relación entre las variables seleccionadas -formación, role model, miedo al fracaso y habilidades percibidas- $y$ si estas influyen sobre la actividad emprendedora siendo esta la variable independiente. Como variables de control se utiliza la edad de la persona, el género y la ubicación geográfica.

\section{Nuevo Emprendedor $r_{i}=b_{0}+b_{1}$ Educación $_{i}$ $+b_{2}$ Ejemplo emprendedor ${ }_{i}+b_{3}$ Miedo al fracaso $_{i}+b_{4}$ Habilidades percibidas $_{i}+b_{5}$ Control $_{i}+e_{i}$ (1)}

En la ecuación (1), $b_{0}$ es la constante del modelo, $b_{n}$ es el vector de parámetros asociados a las variables independientes analizadas, y $e_{i}$ es el error del modelo que sigue una distribución logística.

\section{RESULTADOS Y DISCUSIÓN}

Los resultados del modelo de regresión logística que relaciona la actividad emprendedora reciente con las variables asociadas al capital humano formal e informal se presentan en la tabla 2. Es importante destacar que el ajuste del modelo propuesto es bastante positivo tanto en términos del Pseudo-R2 $(0,1471)$ como en términos del porcentaje de observaciones correctamente clasificadas (70\% para toda la muestra y $72,73 \%$ para el grupo de emprendedores).

Respecto a los resultados de las variables relacionadas con la educación, es importante resaltar que tener un grado de educación secundaria reduce de forma significativa la probabilidad de tomar la decisión de emprender. Este resultado es similar al reportado por Van Praag et al. (2013) y Honjo et al. (2013). Más en concreto, los resultados del efecto marginal medio indican que, en promedio, la probabilidad de crear una nueva empresa entre las personas con educación secundaria es

Tabla 2: Modelo de regresión logística para la actividad emprendedora

\begin{tabular}{|c|c|c|}
\hline & \multicolumn{2}{|c|}{ Emprendedor de reciente creación } \\
\hline & $\begin{array}{l}\text { Coeficiente } \\
\text { (Error Std.) }\end{array}$ & $\begin{array}{l}\text { Efecto marginal } \\
\text { medio }\end{array}$ \\
\hline \multirow{2}{*}{$\begin{array}{l}\text { Ejemplo emprendedor } \\
\text { (Role model) }\end{array}$} & $1,1732^{* * *}$ & $0,0405^{* * *}$ \\
\hline & $(0,2860)$ & $(0,0103)$ \\
\hline \multirow{2}{*}{ Miedo al fracaso } & $-0,8270^{* * *}$ & $-0,0285^{* * *}$ \\
\hline & $(0,3030)$ & $(0,0107)$ \\
\hline \multirow{2}{*}{ Habilidades percibidas } & $1,2240^{* * *}$ & $0,0422^{* * *}$ \\
\hline & $(0,3504)$ & $(0,0107)$ \\
\hline \multirow{2}{*}{ Género } & $-0,6371^{* *}$ & $-0,0220^{* *}$ \\
\hline & $(0,2583)$ & $(0,0090)$ \\
\hline \multirow{2}{*}{ Edad (ln años) } & 0,2903 & 0,0101 \\
\hline & $(0,3262)$ & $(0,0113)$ \\
\hline \multirow{2}{*}{ Educación primaria } & $-0,4628$ & $-0,0160$ \\
\hline & $(0,3307)$ & $(0,0115)$ \\
\hline \multirow{2}{*}{ Educación secundaria } & $-0,7561^{* *}$ & $-0,0261^{* *}$ \\
\hline & $(0,3053)$ & $(0,0106)$ \\
\hline Variables regionales (dummy) & $\mathrm{Si}$ & \\
\hline \multirow{2}{*}{ Constante } & $-4,7219^{* * *}$ & \\
\hline & $(1,2915)$ & \\
\hline Wald test (chi2) & $90,54^{* * *}$ & \\
\hline Log likelihood & $-278,68$ & \\
\hline Pseudo R2 (McFadden) & 0,1471 & \\
\hline $\begin{array}{c}\% \text { Correctamente predicho } \\
\text { (emprendedores) }\end{array}$ & 0,7273 & \\
\hline $\begin{array}{l}\% \text { Correctamente predicho } \\
\text { (no emprendedores) }\end{array}$ & 0,6986 & \\
\hline $\begin{array}{l}\% \text { Correctamente predicho } \\
\text { (muestra total) }\end{array}$ & 0,6997 & \\
\hline Observaciones & 2011 & \\
\hline
\end{tabular}

Nota: los valores entre paréntesis representan el error estándar, ${ }^{*} \mathrm{p}<0,10$;

${ }^{* *} \mathrm{p}<0,05 ;{ }^{* * *} \mathrm{p}<0,01$, San José es la variable regional omitida.

2,61 puntos porcentuales inferior respecto a la probabilidad de emprendimiento entre los individuos con formación post-secundaria (vocacional / universitaria). Estos resultados permiten confirmar nuestra primera hipótesis que propone que el capital humano formal (educación) tiene un efecto positivo sobre la decisión de crear una empresa.

En el caso de la variable de autocon- fianza -habilidad percibida-, se observa que esta variable afecta de forma positiva la actividad emprendedora, concordando con los resultados mostrados por, entre otros, Lafuente y Vaillant (2007), Shook et al. (2003) y Karabay et al. (2016). Para esta variable, se observa que la probabilidad de emprendimiento aumenta 4,22 puntos porcentuales entre quienes manifiestan autoconfianza en 


\section{Se aporta evidencia científica que los ejemplos emprendedores (role model) influyen positivamente en la decisión emprendedora}

sus habilidades emprendedoras, respecto a la probabilidad de quienes manifiestan no tener esta habilidad. La autoconfianza se asocia a la percepción de contar con el conocimiento, las competencias, optimismo, autoafirmación y persistencia para crear y gestionar un negocio. Este resultado ofrece un fuerte y sólido apoyo a la segunda hipótesis que propone que el capital humano informal (autoconfianza) tiene un efecto positivo sobre la decisión de crear una empresa.

Se observa además que tener un role model influye positivamente en la decisión de crear una empresa. Este resultado está en línea con investigaciones previas que analizan esta variable (por ejemplo, Lafuente y Vaillant, 2007; Bosma et al., 2012). Los ejemplos emprendedores no sólo transmiten mensajes positivos sobre el emprendimiento, sino que además pueden facilitar al individuo el descubrir y actuar sobre nuevas ideas y oportunidades de negocios durante las etapas iniciales del proceso emprendedor. La interacción con emprendedores impulsa el aprendizaje y proporciona oportunidades para comprender mejor las tareas asociadas a la gestión empresarial. En este caso, los resultados en la tabla 2 muestran cómo la probabilidad de emprendimiento aumenta 4,05 puntos porcentuales entre las personas que cuentan con un role model, respecto a la probabilidad de quienes no conocen un emprendedor reciente.
Por su parte, el miedo al fracaso ejerce un efecto negativo sobre la actividad emprendedora. En concreto, la probabilidad de crear una nueva empresa entre quienes manifiestan miedo al fracaso disminuye 2,85 puntos porcentuales, respecto a la probabilidad de emprendimiento entre los individuos que no perciben que el miedo al fracaso es un obstáculo al emprendimiento. Este resultado está en línea con investigaciones previas (Lafuente y Vaillant, 2007; Cacciotti, Hayton, Mitchell y Giazitzoglu, 2016).

GEM (por ejemplo, Driga et al., 2009). Además, este resultado está en línea con Zhang et al., (2009), quienes encuentran que ser mujer hace menos probable que se lleve a cabo un emprendimiento secundando.

\section{CONCLUSIONES FINALES Y LÍNEAS FUTURAS DE INVESTIGACIÓN}

Este documento analiza cómo distintas formas de capital humano afectan la decisión emprendedora. Los resultados del análisis empírico muestran que existe una mayor actividad emprendedora en personas que poseen una formación académica alta (vocacional/universitaria). Sin embargo, es importante prestar atención a las estadísticas mostradas por quienes poseen una formación académica menor -primaria y secundaria- de modo que desde las instancias del Gobierno y la academia se creen, revisen o modifiquen las estructuras académicas actuales, con el fin de incentivar y motivar un cambio de mentalidad en la población académica, y como resultado se llegue a poseer una sociedad decidida hacia el emprendimiento que

Finalmente, en cuanto a las variables de control, los resultados indican que la actividad emprendedora es significativamente inferior entre las mujeres. Los resultados de la tabla 2 sugieren que, dentro de la muestra del estudio, la probabilidad de emprendimiento es 2,20 puntos porcentuales superior entre los hombres, en comparación con la probabilidad de emprendimiento de las mujeres. Estos resultados están en línea con estudios previos basados en datos generados por el impacte social y económicamente, y revierta así los indicadores actuales.

Al denotarse una menor intención emprendedora entre las mujeres y quienes poseen una menor formación -primaria y secundaria-, se considera de importancia el crear mecanismos de educación que permitan fomentar el emprendimiento femenino, en los que se destaquen ejemplos emprendedores, los cuales, a pesar de $\gg$ 


\section{Las personas con autoconfianza en sus habilidades emprendedoras -habilidad percibida- deciden emprender más en comparación a otra persona que no cuente con esta habilidad}

\los obstáculos que se les presentan, deciden optar por la creación de empresas, generando un incremento en las tasas de empleo y reduciendo la dependencia particularmente de las zonas rurales hacia el Estado.

La presencia de role model emprendedor en la sociedad causa un efecto positivo en aquellos individuos que consideran al emprendimiento dentro de sus metas personales, sin embargo, se debe hacer un esfuerzo desde la academia, empresa privada e instituciones del Estado involucradas en el apoyo al emprendimiento, por revertir el alto porcentaje que presenta el miedo al fracaso, siendo uno de los mecanismos de reducción del impacto la exposición de ejemplos emprendedores, cuyas enseñanzas y acciones de mejora impulsan los futuros emprendimientos.

Fundamentales han de ser los esfuerzos que se realicen desde la academia, la empresa privada e instituciones del Estado involucrados en el desarrollo del emprendimiento para revertir el alto porcentaje de personas que manifiestan un miedo al fracaso emprendedor como un obstáculo a la actividad emprendedora. Algunas de las maneras de aminorar dicho sentimiento es exaltando, ante la sociedad, la presencia de role model quien, a pesar de las vicisitudes y el panorama que se pueda presentar, posee un alto nivel de autoconfianza que le permite desarrollar emprendimientos que generan empleos, aportan a la economía y promueven el desarrollo social de un país. Es conveniente resaltar, además, la importancia de que para crear un emprendimiento no existe edad, donde la sociedad no discrimina a quien opte por el desarrollo de un emprendimiento, ya que más bien, le incentiva a intentarlo.

Como en todo estudio, existe una serie de limitaciones a destacar que, por otra parte, representan futuras líneas de investigación. En primer lugar, una posible línea de investigación futura podría centrarse en el análisis de la relativamente inferior tasa de actividad emprendedora entre las mujeres costarricenses. Por ejemplo, y a la vista de nuestros resultados en la tabla 2 y el apéndice 3, estudios futuros podrían explorar por qué la actividad emprendedora reciente (baby-business) de las mujeres es inferior a la de los hombres, cuando la actividad emprendedora incipiente (naciente) femenina no es significativamente distinta a la observada en los hombres. En segundo lugar, la investigación futura podría centrarse en el análisis de otras dimensiones -igualmente relevantes- que no fueron evaluadas en el presente estudio, por ejemplo: el efecto conjunto de la edad del individuo con su ubicación geográfica (zona urbana versus zona rural) o la relación entre la auto-confianza y las expectativas de crecimiento empresarial de las nuevas empresas, en términos de empleo.

\section{Referencias bibliográficas}

Amorós, J. E. (2011). El Proyecto Global Entrepreneurship Monitor (GEM): Una Aproximación desde el contexto Latinoamericano. Academia. Revista Latinoamericana de Administración, (46), 1-15.

Arora, P., Haynie, J. M. y Laurence, G. A. (2013). Counterfactual thinking and entrepreneurial self-efficacy: The moderating role of self-esteem and dispositional affect. Entrepreneurship: Theory and Practice, 37(2), 359-385.

Bandura, A. (1999). A social cognitive theory of personality. In L. Pervin \& O. John (Ed.), Handbook of personality (2nd ed., pp. 154-196). New York: Guilford Publications.

Baron, R. (2008). The role of affect in the entrepreneurial process. Academy of Management Review, 33(2), 328-340. Bayon, M. C., Lafuente, E. y Vaillant, Y. (2016). Human capital and the decision to exploit innovative opportunity. Management Decision, 54(7), 1615-1632.

Benabou, R. y Tirole, J. (2002). Self-confidence and personal motivation. Quarterly Journal of Economics, 117 (3), 871-915.

Becker, G.S. (2003). Human capital: A theoretical and empirical analysis with special reference to education. University of Chicago Press, Chicago, IL.
Bell, J., Callaghan, I., Demick, D. y Scharf, F. (2004). Internationalizing Entrepreneurship Education. Journal of International Entrepreneurship, 2(2), 109-124.

Bosma, N., Hessels, J., Schutjens, V., Van Praag, M. y Verheul, I. (2012). Entrepreneurship and role models. Journal of Economic Psychology, 33 (2), 410-424.

Cacciotti, G., Hayton, J. C., Mitchell, J. R. y Giazitzoglu, A. (2016). A reconceptualization of fear of failure in entrepreneurship. Journal of Business Venturing, 31(3), 302-325.

Cardon, M. S. y Kirk, C. P. (2015). Entrepreneurial Passion as Mediator of the Self-Efficacy to Persistence Relationship. Entrepreneurship: Theory and Practice, 39(5), 1027-1050.

Debrulle, J., Maes, J. y Sels, L. (2013). Start-up absorptive capacity: Does the owner's human and social capital matter?. International Small Business Journal, 32(7), 777 801.

Driga, O., Lafuente, E. y Vaillant, Y. (2009). Reasons behind the relatively lower entrepreneurial activity levels of rural women: Looking into rural Spain. Sociologia Ruralis, 49 (1), 70-96.

Edelman, L. F., Manolova, T., Shirokova, G. y Tsukanova, T. (2016). The impact of family support on young entrepreneurs' start-up activities. Journal of Business Venturing, 31(4), 428-448.

Estrin, S., Mickiewicz, T. y Stephan, U. (2016). Human capital in social and commercial entrepreneurship. Journal of Business Venturing, 31(4), 449-467.

Gelaidan, H. M. y Abdullateef, A. O. (2017). Entrepreneurial intentions of business students in Malaysia. Journal of Small Business and Enterprise Development, 24(1), 54-67.

Ley núm. 8262 de Fortalecimiento de las Pequeñas y Medianas Empresas. (2002). Publicada en La Gaceta N ${ }^{\circ} 94$. San José: Imprenta Nacional.

Henley, A. (2007). Entrepreneurial aspiration and transition into self-employment: evidence from British longitudinal data. Entrepreneurship and Regional Development, 19(3), 253-280.

Honjo, Y., Kato, M. y Okamuro, H. (2013). R\&D investment of start-up firms: does founders' human capital matter?. Small Business Economics, 1-14.

Indrawati, N. K., Salim, U., Djumahir, A. y Djawahir, H. (2015). Moderation Effects of Entrepreneurial Self-efficacy in Relation between Environmental Dimensions and Entrepreneurial Alertness and the Effect on Entrepreneurial Commitment. Procedia - Social and Behavioral Sciences, 169, 13-22.

Jayawarna, D., Jones, O. y Macpherson, A. (2014). Entrepreneurial potential: The role of human and cultural capitals. International Small Business Journal, 32(8), 918919.

Kakouris, A. (2016). Exploring entrepreneurial conceptions, beliefs and intentions of Greek graduates. In- $\quad 》$ 


\section{APÉNDICE}

Apéndice 1: Resumen de la revisión de la literatura: Emprendimiento y capital humano formal

\begin{tabular}{l}
\multicolumn{1}{c}{ Artículo } \\
\hline \\
Unger, Rauch, Frese \\
y Rosenbusch \\
(2011) \\
\\
Kato y Honjo \\
$(2015)$
\end{tabular}

Kakouris

(2016)

Ucbasaran, Westhead y Wright (2008)

Robinson y Sexton (1994)

\begin{tabular}{c} 
Objetivos \\
\hline \\
Identificar estudios empíricos que \\
coincidan con los moderadores \\
teóricos derivados de esta relación \\
que se refieren a conceptualizaciones \\
del Capital Humano $(\mathrm{CH})$, al \\
contexto y a la medición del éxito.
\end{tabular}

Estimar la probabilidad de que un nuevo emprendimiento se retire del sector, identificando los factores que determinan su salida.

Identificar las concepciones, creencias emprendedoras y los determinantes en la intención emprendedora, comparando graduados de ciencias y economía con los emprendedores nacientes $y$ emprendedores en general.

Identificar la relación entre el perfil del emprendedor en capital humano y la búsqueda de oportunidades.

Examinar la relación entre la educación formal, la experiencia y el autoempleo, así como otras variables moderadoras relacionadas con la vida de un individuo y su experiencia.

\section{Método}

Meta-análisis de los moderadores teóricos derivados de la relación entre el capital humano y el éxito empresarial.

Modelo de estimación tiempo/ riesgo para determinar la supervivencia de los nuevos emprendimientos como desempeño post ingreso. Así como un modelo de duración discreta para examinar los

factores que afectan la duración del emprendimiento y cómo varían según la ruta de salida.

Modelo de ecuaciones estructurales aplicado a un cuestionario de 34 ítems.

Las preguntas se refieren a cinco temas principales del pensamiento emprendedor:

conceptualización del emprendimiento, factores de éxito empresarial, motivación, gestión del riesgo y financiación empresarial. La intención emprendedora se identifica a través de una escala de seis ítems.

Aplicación de un cuestionario estructurado, utilizando el muestreo por cuotas a cuatro industrias (agricultura, silvicultura y pesca, producción, construcción y servicios). Se emplea un análisis logístico y regresión probabilística de mínimos cuadrados para probar las hipótesis.

Modelos de regresión lineal múltiple y una técnica de regresión no lineal (probit).

\section{Resultado}

Existe una relación positiva entre el capital humano y el éxito. Los resultados son mayores para las inversiones de capital humano conocimiento/habilidades- que para las inversiones de capital humano -educación/ experiencia-, así como capital humano con alta relación con las tareas en comparación con la baja relación con las tareas, y para las empresas jóvenes en comparación con las empresas de más edad.

El capital humano es medido como educación, y es importante para reducir la probabilidad de quiebra en sectores de alta tecnología. Emprendedores con alto capital humano tienen más probabilidad de cerrar voluntariamente las empresas en sectores de alta y baja tecnología.

Los graduados en ciencias y economía exhiben diferencias en sus creencias, reduciendo la brecha a través de la educación emprendedora. Los emprendimientos incipientes están respaldados por competencias personales, autoconfianza, capacidad de planificación y adopción de un estilo emprendedor.

Las personas con estudios de posgrado, tienen menor probabilidad de identificar más oportunidades, en relación a personas que tienen educación básica. Los emprendedores con altos niveles de capital humano identifican más oportunidades de negocio. El capital humano específico explica la variación en las oportunidades de negocios identificadas y perseguidas, con respecto al capital humano general.

La educación general tiene una influencia positiva sobre el espíritu emprendedor. 


\begin{tabular}{|c|c|}
\hline Artículo & Objetivos \\
\hline $\begin{array}{l}\text { Kirkwood } \\
\text { (2009) }\end{array}$ & $\begin{array}{l}\text { Explorar cómo la confianza en } \\
\text { sí mismos de mujeres y hombres } \\
\text { impactó en la decisión de la } \\
\text { puesta en marcha de sus negocios. } \\
\text { Comprender cómo la confianza en sí } \\
\text { mismos influye en los emprendedores } \\
\text { y el emprendimiento. Identificar y } \\
\text { explicar las diferencias de género en } \\
\text { los niveles de autoconfianza en cada } \\
\text { una de estas dos etapas. }\end{array}$ \\
\hline $\begin{array}{l}\text { Karabay, Akyüz } \\
\text { y Elçi } \\
(2016)\end{array}$ & $\begin{array}{c}\text { Examinar la interacción entre } \\
\text { el conflicto trabajo-familia, el } \\
\text { lugar del control, la confianza en } \\
\text { sí mismo y la personalidad de la } \\
\text { extraversión en los factores de estrés } \\
\text { laboral. }\end{array}$ \\
\hline
\end{tabular}

Gelaidan y

Abdullateef

(2017)

Pihie y Bagheri

(2011)

Sullivan y Marvel

(2011)

Examinar los efectos del apoyo relacional, el apoyo educativo $y$ la confianza en las intenciones emprendedoras de estudiantes de negocios de pregrado en una universidad en Malasia.

Examinar la autoconfianza emprendedora percibida de los profesores y estudiantes de secundaria técnica y vocacional, y si hay una diferencia significativa entre la autoconfianza emprendedora de los profesores y los estudiantes.

Examinar los factores relacionados con el número de trabajadores empleados en las

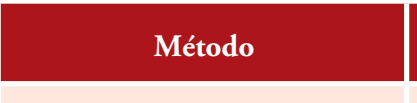

Estudio cualitativo basado en entrevistas a 50 empresarios -25 hombres y 25 mujeres- , cuya muestra surge del New Zealand Business Who's Who, un listado de empresas y sus propietarios, a los cuales se les entrevistó cara a cara por parte del investigador, quien utilizó el paquete de software QSR NUD*IST Vivo (Nvivo) para la administración de los datos.

Investigación cuantitativa, donde los datos fueron recogidos a través del cuestionario, utilizando el programa de paquetes estadísticos SPSS, para examinar los datos recopilados. La muestra total de este estudio fue de 413 de 64 instituciones diferentes. PYME en los Estados Unidos.
Método cuantitativo de recolección y análisis de datos. El tamaño de la muestra para este estudio fue de 364 estudiantes de la Universidad de Utara en Malasia. Se utilizó el modelo de ecuaciones estructurales utilizando el software SmartPLS.

Diseño descriptivo, en el cual se utilizó un instrumento de autoconfianza emprendedora para medir este aspecto en los profesores y estudiantes de secundaria técnica y vocacional.

Estudio cuantitativo, aplicado a empresas PYME, que poseen menos de 500 trabajadores, la información se extrae por el empleo de encuestas.

\section{Resultado}

Las mujeres muestran una falta de confianza en sus capacidades emprendedoras en comparación con los hombres. Una vez establecido un negocio, las mujeres se relacionan con el espíritu emprendedor menos que los hombres. Para algunas mujeres, la autoconfianza emprendedora creció a lo largo del tiempo en el negocio; para otras, parece que continúa actuando como una limitación que afecta su capacidad para acceder a las finanzas y reducir sus aspiraciones de crecimiento.

El conflicto trabajo-familia afecta la sobrecarga de trabajo, y crea un pobre ambiente laboral, y son mecanismos estresantes. El locus de control interno es afectivo sobre la mala congruencia del papel y la estructura organizacional pobre negativamente, mientras que el locus externo del control es afectivo en la estructura organizativa pobre positivamente.

La intención emprendedora de los estudiantes de negocios está influenciada por el apoyo educativo y relacional; sin embargo, los efectos moderadores de la autoconfianza en la relación entre el apoyo educativo, el apoyo relacional ,y la intención empresarial no son significativos. Los apoyos relacionales y educativos son factores importantes que influyen en la intención empresarial de los estudiantes universitarios.

Existe una diferencia significativa entre la autoconfianza emprendedora de profesores y estudiantes. Se requiere, además, de intervenciones más efectivas en términos de enseñanza y aprendizaje emprendedor, que aseguren la implementación exitosa de "el emprendimiento como carrera alternativa"

para todos los estudiantes técnicos y vocacionales.

Existe una relación positiva entre la amplitud del conjunto de conocimientos de un emprendedor y el número de trabajadores, donde la cantidad de vínculos -red-modera positivamente la relación entre el conjunto de conocimientos del emprendedor y el número de empleados. 
Apéndice 3: Modelo de regresión logística para la actividad emprendedora para emprendedores nacientes

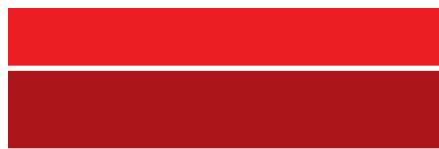

\section{Emprendedor naciente}

Coeficiente

(Error Std.)

Efecto marginal

Ejemplo emprendedor (Role model)

$0,7105^{* * *}$
medio

(Role model)

Habilidades percibidas

Género

Edad (ln años)

Educación primaria

Educación secundaria

Variables regionales (dummy)
Constante

\begin{tabular}{|c|}
\hline Wald test (chi2) \\
\hline Log likelihood \\
\hline Pseudo R2 (McFadden) \\
\hline$\%$ Correctamente predicho \\
(emprendedores)
\end{tabular}

\% Correctamente predicho
(no emprendedores)

\% Correctamente predicho (muestra total)

Observaciones

10,

$(0,1789)$

1

$0,0473^{* * *}$

$-0,4468^{* *}$

$(0,1980)$

$(0,0120)$

$1,7525^{* * *}$

$(0,2818)$

$-0,0297^{* *}$

$(0,0132)$

0,

0,0578

$(0,1738)$

$\mathbf{0 , 0 5 0 5}$

$0,1166^{* * *}$

$(0,0197)$

$(0,2391)$

$\mathbf{0 , 0 0 3 8}$

(0,

0,0021

$(0,2407)$

0,0219

$(0,1985)$

$(0,0116)$

\begin{tabular}{|c|c|}
\hline$(0,1985)$ & $(0,0162)$ \\
\hline$-4,0369^{* * *}$ & \\
\hline$(0,8859)$ & \\
\hline $75,21^{* * *}$ & \\
\hline$-487,11$ & \\
\hline 0,0998 & \\
\hline 0,7908 & \\
\hline 0,5893 & \\
\hline 0,6047 & \\
\hline 2011 & \\
\hline
\end{tabular}

Nota: los valores entre paréntesis representan el error estándar, ${ }^{*} \mathrm{p}<0,10$; ${ }^{* *} \mathrm{p}<0,05 ;{ }^{* * *} \mathrm{p}<0,01$. San José es la variable regional omitida.

$\gg$

ternational Journal of Entrepreneurial Behavior \& Research, 22(1), 109-132.

Kantis, H., Federico, J. y García, S. I. (2014). Indice de Condiciones Sistémicas para el Emprendimiento Dinámico. Rafaela: Asociación Civil Red Pymes Mercosur.

Karabay, M. E., Akyüz, B. y Elçi, M. (2016). Effects of Family-Work Conflict, Locus of Control, Self Confidence and Extraversion Personality on Employee Work Stress. Procedia - Social and Behavioral Sciences, 235(Octubre), 269-280.

Kato, M. y Honjo, Y. (2015). Entrepreneurial human capital and the survival of new firms in high- and lowtech sectors. Journal of Evolutionary Economics, 25(5), 925-957.
Kirkwood, J. (2009). Is a lack of self-confidence hindering women entrepreneurs? International Journal of Gender and Entrepreneurship, 1(2), 118-133.

Lafuente, E., Szerb, L. y Acs, Z.J. (2016). Country level efficiency and national systems of entrepreneurship: A data envelopment analysis approach. Journal of Technology Transfer, 41(6), 1260-1283.

Lafuente, E., Vaillant, Y. y Rialp J. (2007). Regional differences in the influence of Role-Models: Comparing the Entrepreneurial Process of Rural Catalonia. Regional Studies, 41(6), 779-796.

Marvel, M. y Lumpkin, G. (2007). Technology entrepreneurs' human capital and its effects on innovation radicalness. Entrepreneurship: Theory and Practice, 31(6), 807-828.
Marvel, M. R., Davis, J. L. y Sproul, C. R. (2014). Human Capital and Entrepreneurship Research: A Critical Review and Future Directions. Entrepreneurship Theory and Practice, (315).

Ministerio de Ciencia Tecnología y Telecomunicaciones. (2007). Atlas para la Innovación en Costa Rica, 1.

Delmar, F. y Holmquist, C. (2004). Promoting Entrepreneurship and Innovative SMEs in a Global Economy. 2nd OECD Conference of Ministers responsible for SMEs, Istanbul, 3-5 June.

Pihie, Z.A.L. y Bagheri, A. (2011). Teachers' and students' entrepreneurial self-efficacy: Implication for effective teaching practices. Procedia - Social and Behavioral Sciences, 29, 1071-1080.

Programa Estado de la Nación en Desarrollo Humano Sostenible (Costa Rica) PEN. (2014). Estado de la Ciencia, la Tecnología y la Innovación. San José: EDISA S.A.

Robinson, P. B. y Sexton, E. A. (1994). The effect of education and experience on self-employment success. Journal of Business Venturing, 9(2), 141-156.

Shane S. (1996). Explaining variation in rates of entrepreneurship in the United States: 1899-1988. Journal of Management, 22(5), 47-781.

Shook, C. L., Priem, R. L. y McGee, J. E. (2003). Venture creation and the enterprising individual: A review and synthesis. Journal of Management, 29(3), 379-399.

Sullivan, D. y Marvel, M. (2011). How Entrepreneurs' Knowledge and Network Ties Relate to the Number of Employees in New SMEs. Journal of Small Business Management, 49(2), 185-206.

Ucbasaran, D., Westhead, P. y Wright, M. (2008). Opportunity identification and pursuit: Does an entrepreneur's human capital matter?. Small Business Economics, 30(2), 153-173.

Unger, J. M., Rauch, A., Frese, M. y Rosenbusch, N. (2011). Human capital and entrepreneurial success: A meta-analytical review. Journal of Business Venturing, 26(3), 341-358.

Van Praag, M., van Witteloostuijn, A. y van der Sluis, J. (2013). The higher returns to formal education for entrepreneurs versus employees. Small Business Economics, 40(2), 375-396.

Vaillant, Y. y Lafuente, E., (2007). Do different institutional frameworks condition the influence of local fear of failure and entrepreneurial examples over entrepreneurial activity? Entrepreneurship y Regional Development, 19(4), 313-337.

Westhead, P., Ucbasaran, D. y Wright, M. (2009). Information Search and Opportunity Identification: The Importance of Prior Business Ownership Experience. International Small Business Journal, 27(6), 659-680.

World Bank. (2014). Doing Business 2014 Measuring Regulatory Quality and Efficiency. Doi: 10.1596/978-14648-0667-4.

Zhang, Z., Zyphur, M. J., Narayanan, J., Arvey, R. D., Chaturvedi, S., Avolio, B. J. y Larsson, G. (2009). The genetic basis of entrepreneurship: Effects of gender and personality. Organizational Behavior and Human Decision Processes, 110(2), 93-107. T 